\section{Dexmedetomidine-associated fever in the intensive care unit}

\author{
Andrew C. Faust and Shelby E. Sutton
}

\author{
Ther Adv Drug Saf \\ 2015, Vol. 6(6) 234-237 \\ DOI: $10.1177 /$ \\ 2042098615612661 \\ (C) The Author(s), 2015 \\ Reprints and permissions: \\ http://www.sagepub.co.uk/ \\ journalsPermissions.nav
}

\section{Introduction}

Dexmedetomidine is an intravenous, $\alpha-2$ receptor agonist commonly used for the maintenance of sedation in the intensive care unit (ICU) [Hospira, 2014] (Box 1). The most recent iteration of the Society of Critical Care Medicine guidelines for pain, agitation and delirium preferentially recommend using a nonbenzodiazepine agent, such as dexmedetomidine, if a continuousinfusion sedative is required [Barr et al. 2013]. This recommendation is based on multiple trials which have demonstrated the benefits of dexmedetomidine over benzodiazepine infusions in mechanically ventilated ICU patients [Riker $e t$ al. 2009; Pandharipande et al. 2007; Jakob et al. 2012]. There is limited respiratory depression and the potential for less ICU-related delirium associated with the drug, which makes it an attractive option for ICU patients. Additionally, dexmedetomidine has demonstrated safety and efficacy outside of the mechanically ventilated patient and has been evaluated in situations such as alcohol withdrawal [Mueller et al. 2014].

The most commonly reported adverse effects associated with dexmedetomidine are hypotension and bradycardia, which can result in sinus arrest [Hospira, 2014]. Fever, or pyrexia, has been reported with dexmedetomidine use; however, this rate was no different than the control arm (4-5\% for both dexmedetomidine and control) [Hospira, 2014]. In a more recent publication comparing midazolam or propofol with dexmedetomidine, there were no differences in the incidence of fever [Jakob et al. 2012]. Two case reports have been published describing high fevers related to dexmedetomidine [Okabe et al. 2009; Reeve et al. 2013] As fever in the ICU is commonly attributed to infections, underlying malignancy or thrombosis, development of an elevated temperature may prompt collection of multiple cultures, laboratory tests, imaging studies and other diagnostic testing. Although drug fever is typically a diagnosis of exclusion and should not subvert the need for common tests (e.g. blood cultures), timely recognition of druginduced causes may avoid more invasive, costly or extensive interventions.

In this article, we describe a patient who developed temperatures up to $40.3^{\circ} \mathrm{C}$ after initiation of dexmedetomidine, which completely resolved after discontinuation of the drug.

\section{Case report}

The patient is a 57-year old female admitted to our facility for an acute exacerbation of chronic
Correspondence to: Andrew C. Faust, PharmD, BCPS

Texas Health Presbyterian Hospital of Dallas, Dallas,

TX 75231, USA

andrewfausta

texashealth.org

Shelby E. Sutton, MD Southwest Pulmonary Associates - Pulmonary/ Critical Care Medicine,

Dallas, TX, USA

Box 1. Key points about dexmedetomidine.

- Dexmedetomidine (Precedex ${ }^{\circledR}$, Hospira, Inc.) is a commonly used sedative agent in intensive care and operative settings for maintaining a lighter level of sedation.

- Although some investigators have evaluated $\alpha 2$-agonists as a potential target for the treatment of hyperthermia, fever has previously been reported with this agent. In our case and the previous published cases, this fever is quite impressive and may prompt investigation of causes such as neuroleptic malignant syndrome.

- Withdrawal of dexmedetomidine results in defervescence within a few hours of cessation of the infusion. 
obstructive pulmonary disease (COPD) and altered mental status. Her past medical history was pertinent for emphysema requiring home oxygen therapy and an unspecified anxiety disorder. She was a current smoker and used methamphetamine recreationally, with her last use approximately 2 weeks prior to arrival. Prior to admission, her medications included clonazepam $6 \mathrm{mg}$ per day, trazodone $500 \mathrm{mg}$ at bedtime, quetiapine extended release $600 \mathrm{mg}$ at bedtime and ziprasidone $80 \mathrm{mg}$ daily, although there were concerns with noncompliance with her prescription regimen.

Upon arrival to our facility, she was intubated and transferred to the ICU. Methylprednisolone and levofloxacin were initiated for acute COPD exacerbation. Given the patient's polysubstance use and ventilator requirements, she was sedated with both fentanyl and propofol with a goal Richmond Agitation-Sedation Scale score (RASS) of 0 to -2 . Over the subsequent 48 hours, she remained agitated (RASS +2 to +4 ) despite high doses of sedation and analgesia. Quetiapine was restarted at $100 \mathrm{mg}$ twice daily and was increased after 24 hours to $200 \mathrm{mg}$ twice daily. As her ventilator needs lessened, a dexmedetomidine infusion was started in an attempt to keep the patient calm enough to successfully extubate. Dexmedetomidine was initiated at $0.2 \mu \mathrm{g}$ per $\mathrm{kg}$ per hour without a bolus due to the patient's fluctuating heart rate (rates of $50-80$ beats per minute) in an attempt to curb iatrogenic bradycardia.
The medication was increased by $0.1 \mu \mathrm{g}$ per $\mathrm{kg}$ per hour every 45-60 minutes for a target RASS of 0 to +2 . She remained calm but slightly agitated (RASS +1 to +2 ) on a dose of $0.3-0.5 \mu \mathrm{g}$ per kg per hour and was liberated from mechanical ventilation on hospital day 5 .

Approximately 24 hours after initiation of dexmedetomidine, she developed a fever of $38.1^{\circ} \mathrm{C}$. Her temperature continued to increase over the following 24 hours, despite 2 administrations of intravenous acetaminophen. A period of 36 hours after the start of dexmedetomidine, her temperature remained above $39^{\circ} \mathrm{C}$ and cultures were obtained (Figure 1). She was administered a single dose of vancomycin and remained on levofloxacin, but no additional antibiotics were added. Her cultures remained sterile, urinalysis did not reveal signs of infections, and there were no acute changes on her chest radiograph to suggest a new infection. The patient remained on dexmedetomidine $0.3-0.5 \mu \mathrm{g}$ per kg per hour until approximately 72 hours after initiation. At this point, the decision was made to stop dexmedetomidine as it might have been the source of fever. The drug was titrated off over the next 5 hours; 3 hours after discontinuation of the dexmedetomidine, the patient became afebrile for the first time in approximately 56 hours. Aside from the two doses of intravenous acetaminophen, she was not administered any other antipyretic medications during this time period. Quetiapine was continued and intravenous lorazepam $2 \mathrm{mg}$ was

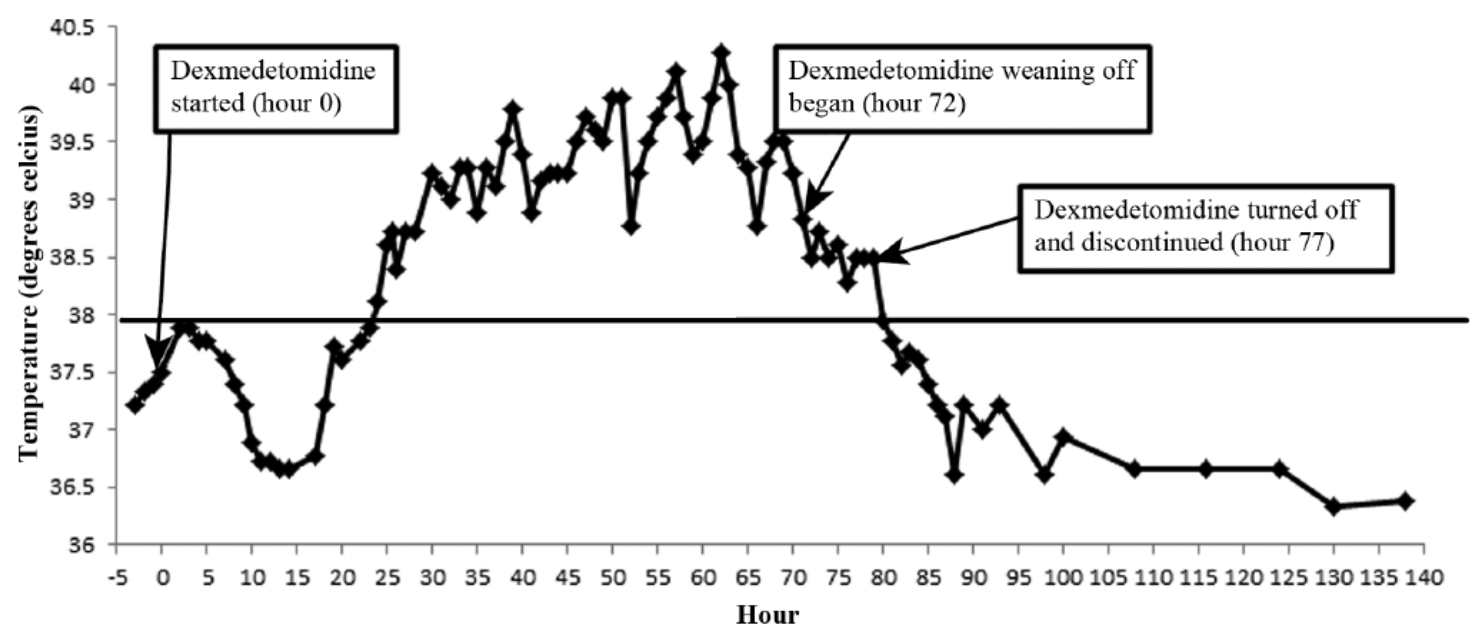

Figure 1. Temperature trend during dexmedetomidine infusion.

Dexmedetomidine was initiated at $0.2 \mu \mathrm{g}$ per $\mathrm{kg}$ per hour at time 0 . The patient developed her first febrile event 24 hours after initiation of the medication. This temperature peaked at $40.3^{\circ} \mathrm{C}$ and was unresponsive to antipyretic medication administration. Dexmedetomidine was weaned down and discontinued starting 72 hours after initiation. She was afebrile 3 hours after permanent discontinuation of the drug. The patient was not rechallenged.

Bold line $=38^{\circ} \mathrm{C}\left(100.4^{\circ} \mathrm{F}\right)$. 
administered as needed for acute agitation. The patient was transferred to the general medical floor and remained afebrile for the remainder of her hospitalization.

\section{Discussion}

Fever attributed to dexmedetomidine has been uncommonly reported, but is a side effect critical care providers should be aware of. As patients in the ICU often receive multiple therapeutic interventions, it is often difficult to assign causation of fever to a single drug agent. In our case report, the temporal relationship of the patient's temperature response and the use of dexmedetomidine would strongly implicate the drug. There were no other identifiable causes of fever in this patient. The patient was a recreational user of methamphetamines; however, her last use was approximately 14 days prior to arrival. Fever does not appear to be a typical symptom of methamphetamine withdrawal and the timeframe does not appear to support this as a mechanism for her fever [Zorick et al. 2010]. Although she received a single dose of vancomycin during her febrile episode, infection was ruled out and all her cultures remained sterile. Her exam ruled out seizures and her thyroid function was within normal limits. Autoimmune testing was not pursued. Although the patient was receiving quetiapine, she displayed no signs or symptoms of neuroleptic malignant syndrome. Additionally, quetiapine was continued with remission of the fever despite ongoing use. Based on this information, we would assign a Naranjo score of 5, making this a 'probable' adverse reaction to dexmedetomidine [Naranjo et al. 1981].

Prior to this case, there have only been two case reports which implicate dexmedetomidine as the cause of fever [Okabe et al. 2009; Reeve and Cooper, 2013]. In the first reported case of dexmedetomidine-induced fever, Okabe and colleagues describe development of fever 22 hours after initiation of the drug at a dose of $0.5-0.7 \mu \mathrm{g}$ per kg per hour. The medication was temporarily discontinued to allow the patient to fully arouse in anticipation of endotracheal extubation. However, when the medication had to be resumed, the patient developed fevers up to $40.6^{\circ} \mathrm{C}$. Dexmedetomidine was stopped and the patient's temperature returned to normal approximately 4 hours later. The authors did a thorough job of ruling out other causes of fever, including infection. The patient was also administered anti-inflammatory drugs and dantrolene without effect on the temperature. In the second published case, Reeve and Cooper described a 68-year-old male who developed a temperature of $39.4^{\circ} \mathrm{C}$ approximately 8 hours after initiation of a dexmedetomidine $0.8 \mu \mathrm{g}$ per kg per hour infusion. Despite acetaminophen, ice packs and a cooling blanket, the patient remained febrile. His dexmedetomidine was stopped and his fever dissipated after approximately 4 hours. This case was confounded by supratherapeutic lidocaine levels; however, the authors argued that due to the temporal relationship, dexmedetomidine was the more likely causative agent. These authors assigned a Naranjo score of 3 ('possible') to their case.

Additionally, there have been a small number of reports filed with the US Food and Drug Administration (FDA). These cases have been summarized, although details of the cases were limited [Okabe et al. 2009]. Fever or hyperpyrexia was reported in up to $7 \%$ in clinical trials and is summarized in the drug's package labeling. Both this information and the most recent data indicate no difference in development of fever between dexmedetomidine treated patients and those receiving another sedative agent [Hospira, 2014; Jakob et al. 2009].

Similar to the other two published cases, our patient developed a high fever which could not be explained by another cause, such as infection. Our patient developed a fever 24 hours after initiation of a low-dose dexmedetomidine infusion. This was similar to what was described in the first case where the patient received 0.5-0.7 $\mu \mathrm{g}$ per $\mathrm{kg}$ per hour [Okabe et al. 2009]. There may some element of dose response, as the second case developed fever much sooner while receiving 0.8 $\mu \mathrm{g}$ per kg per hour [Reeve and Cooper, 2013].

It is not known why patients would develop fevers while receiving dexmedetomidine. Alpha-2 agonists have been studied and implicated as a potential therapeutic target for hyperthermia and temperature control [Madden et al. 2013]. Animal studies have demonstrated that both dexmedetomidine and clonidine administration result in reductions to the shivering and temperature response to prostaglandin- and toxin-induced fevers. In some cases, this has resulted in hypothermia. It has been proposed that hyperthermia may be an allergic reaction to the medication, although there are no data to substantiate that claim [Okabe et al. 2009]. 


\section{Conclusion}

Fever related to dexmedetomidine appears to be a relatively rare effect of the medication. Clinicians should be aware of this adverse effect, as the fevers in excess of $40^{\circ} \mathrm{C}$ were observed in this report and the other two published cases. Discontinuation of dexmedetomidine results in marked defervescence within several hours.

\section{Funding}

This research received no specific grant from any funding agency in the public, commercial, or notfor-profit sectors.

\section{Conflict of interest statement}

The authors declare no conflicts of interest in preparing this article.

\section{References}

Barr, J., Fraser, G., Puntillo, K., Ely, E., Gelinas, C., Dasta, J. et al. (2013) Clinical practice guidelines for the management of pain, agitation, and delirium in adult patients in the intensive care unit. Crit Care Med 41: 263-306.

Jakob, S., Ruokonen, E., Grounds, R., Sarapohja, T., Garratt, C., Pocock, S. et al. (2012)

Dexmedetomidine vs. midazolam or propofol for sedation during prolonged mechanical ventilation: two randomized controlled trials. FAMA 307: 1151-1160.

Hospira (2014) Precedex $\AA$ (dexmedetomidine hydrochloride) [package insert]. Lake Forest, IL: Hospira, Inc.
Madden, C., Tupone, D., Cano, G. and Morrison, S. (2013) Alpha-2 adrenergic receptor-mediated inhibition of thermogenesis. F Neurosci 33: 2017-2028.

Mueller, S., Preslaski, C., Kiser, T., Fish, D., Lavelle, J., Malkoski, S. et al. (2014) A randomized, double-blind, placebo-controlled dose range study of dexmedetomidine as adjunctive therapy for alcohol withdrawal. Crit Care Med 42: 1131-1139.

Naranjo, C., Busto, U., Sellers, E., Sandor, P., Ruiz, I., Roberts, E. et al. (1981) A method for estimating the probability of adverse drug reactions. Clin

Pharmacol Ther 30: 239-245.

Okabe, T., Takeda, S., Akada, S., Hongo, T. and Sakamoto, A. (2009) Postoperative intensive care unit drug fever caused by dexmedetomidine. Anesth Analg 108: 1589-1591.

Pandharipande, P., Pun, B., Herr, D., Maze, M., Girard, T., Miller, R. et al. (2007) Effect of sedation with dexmedetomidine vs. lorazepam on acute brain dysfunction in mechanically ventilated patients: the MENDS randomized controlled trial. $\mathcal{F} A M A 298$ : 2644-2653.

Reeve, A. and Cooper, B. (2013) Dexmedetomidineinduced fever. F Pharm Tech 29: 215-218.

Riker, R., Shehabi, Y., Bokesch, P., Ceraso, D., Wisemandle, W., Koura, F. et al. (2009) Dexmedetomidine vs. midazolam for sedation of critically ill patients: a randomized trial. $\mathcal{F} A M A 301$ : 489-499.

Zorick, T., Nestor, L., Miotto, K., Sugar, C., Hellemann, G., Scanlon, G. et al. (2010) Withdrawal symptoms in abstinent methamphetamine-dependent subjects. Addiction 105: 1809-1818.
Visit SAGE journals online http://taw.sagepub.com

@SAGE journals 